\title{
Improvement in Coding Time of Embedded Zero Wavelet Tree
}

\author{
Akhilendra Yadav \\ Deptt. of Electrical Engg. \\ COER, Roorkee
}

\author{
M. A. Ansari \\ Deptt of Electrical Engg \\ Gautam Buddha University \\ Greater Noida (UP)
}

\author{
Manoj Tripathy \\ , Deptt of Electrical Engg. \\ IIT,Roorkee
}

\begin{abstract}
As the coming era of digitized information. The Compression is one of the indispensable techniques to solve this problem. Quality and time are two important aspects. Achieving high quality necessarily requires higher degree of skill, sophisticated design tools, advancement. The EMBDDED ZEROTREE WAVELET (EZW) algorithm, as presented by J. Shapiro, is a simple yet powerful algorithm, in which bit-streams are generated in the order of their significance in containing the image information. The original EZW algorithm scans the entire wavelet decomposed image, at a stroke, during each pass. Improvement of quality results reduction in productivity and vice versa. Thus, optimality must be maintained between quality as well as productivity. This work presents a modified method for coding images using EZW method, which works on the principal of fragmentation of the colored image. The proposed method takes the smallest unit cell, generated from the wavelet decomposed image, to encode at a time .This makes the encoding times independent of the level of wavelet decomposition. This work shows advancement in the original work of unit embedded zero tree coding.This paper is based on encoding time for color image processing while the UEZW was totally based on grayscale image. Results show that the proposed algorithm is more efficient in performance, in terms of encoding times, as compared to the original algorithm.
\end{abstract}

\section{Keywords}

Compression, EZW, zero trees.

\section{INTRODUCTION}

Wavelet based image compression techniques are increasingly used for image compression. Wavelets are tools for decomposing signals such as images into a hierarchy of increasing resolutions as we consider more and more detailed look at the image. As a basic tool for decomposing signals, wavelet can be considered as duals to the more traditional Fourier based analysis methods. "If coefficients of a wavelet at one scale in part of the image is not significant (i.e. close to zero), then the higher resolution wavelet coefficients in the same part of the image are also likely to be insignificant. In this paper we are going to study an embedded wavelet coding technique known as unit embedded zero tree wavelet coding that effectively exploits the self-similarity between sub-band and the fact that the high frequency sub-bands mostly contain insignificant coefficients. This zero tree technique takes advantage of hierarchical structure of the wavelet decomposed sub images, using the parent-child-grandchild relationship for compression. The Embedded Zero tree Wavelet (EZW) is based on 4 key concepts: - 1) A Discrete Wavelet Transform or hierarchical decomposition. 2) Prediction of the absence of significant information across scales b exploiting the self similarity inherent in images. 3) Entropy-Coded successive approximation quantization \& 4) Universal lossless data compression which is achieved via adaptive arithmetic coding. Using an embedded code, a coder can terminate the encoding at any desired step taking into account a desired parameter like bitrate, as against the image quality. Similarly, for a given bit stream, the decoder can stop decoding at any step thereby producing reconstructed image corresponding to a lower-rate encoding. Optimally, for a given bit-rate, the non-embedded code must be more efficient than the embedded code, as it is free from those constraints, which are imposed by the embedded coding. The EZW algorithm gives a good performance in terms of time taken for encoding, when relatively images of smaller dimensions are coded using it. However, as the image dimensions increases the time taken for encoding goes on increasing with it.

\section{EMBEDDED ZERO TREE CODING OF WAVELET COEFFICIENTS}

\subsection{Embedded Zero Tree Wavelet Compression}

EZW is based on two hypotheses- first is that if a wavelet coefficient at the location of the coarse level is insignificant with respect to the given threshold $\mathrm{T}$, then all the wavelet coefficient at the same location at the higher level are most likely to insignificant with respect to the same threshold. This hypothesis determines the ZERO TREE structure. The second hypothesis is that the larger wavelet coefficient is more important than the smaller one. There are several ways wavelet transforms can decompose an image into various subbands. Here we have used 2-D wavelet transform .An $\mathrm{M} * \mathrm{~N}$ image is decomposed using wavelet transform .The 2-D wavelet transform the image into four sub-images using after passing a high pass filter and low pass filter which are applied along the rows and then along the columns. These four subbands are called as LOW-LOW, HIGH-LOW, LOW-HIGH and HIGH-HIGH as shown in Fig. (1). The image of low frequency sub-band contains major information. The values of high frequency sub-band approximate to zero. The filters used for obtaining the discrete wavelet transform are based on Daubechies

filters. 


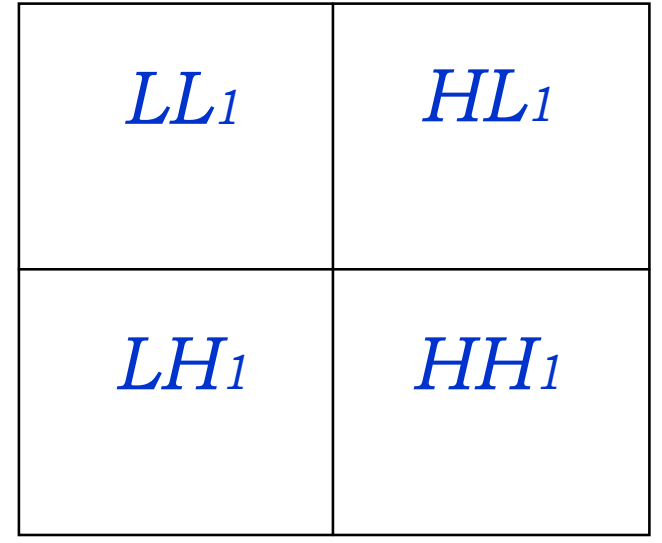

Figure 1. First step of a Discrete wavelet transform: The image is divided into four sub-bands using seperable filters.

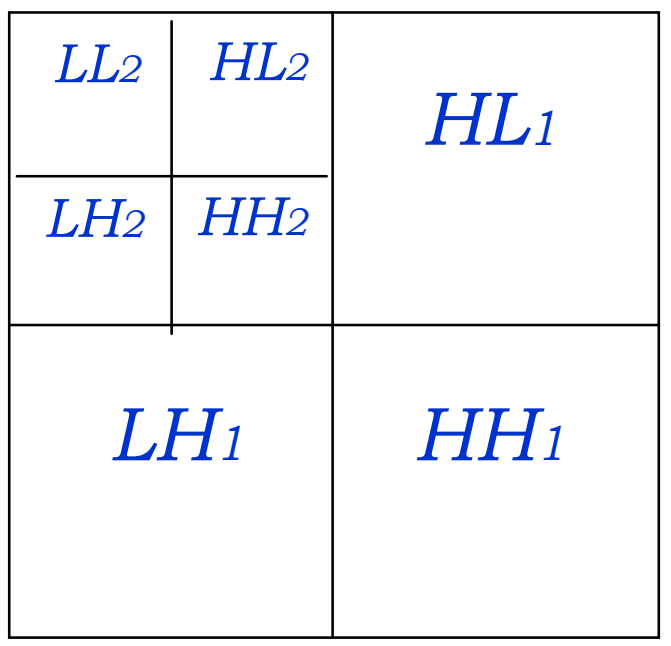

Figure 2. Two scale wavelet decomposition

More specifically, in a hierarchical sub-band system with the expectation of highest frequency sub-bands every coefficient at a given scale can be related to a set of coefficients at the next finer scale of similar orientation. The coefficient at the coarse level is called "parent" and the coefficient at the finer scale in the same orientation is called as "child". For a given parent, the set of each coefficients, at each finer scale having similar orientation and spatial location known as descendants .Similarly for a given child, the coefficients at the coarser scales of same orientation linked to the same location are called ancestors.

\subsection{Concept of Wavelet Coefficient}

A wavelet transform transforms a signal from the time domain to the joint time-scale. To compress the transformed signal, not only the coefficient values, but also there position in time has to be coded. A coefficient in the lower sub-band can be thought of as having four descendants in the next higher subband. Thus each coefficient in the parent band is linked to four coefficients in the child sub-bands. This linked structure is known as the QUAD-TREE .In a zero tree all the coefficients in a quad tree are smaller than the threshold if the parent coefficient is smaller than this threshold .A scanning of the coefficient is performed in such a way that no child coefficient is scanned before its parent.

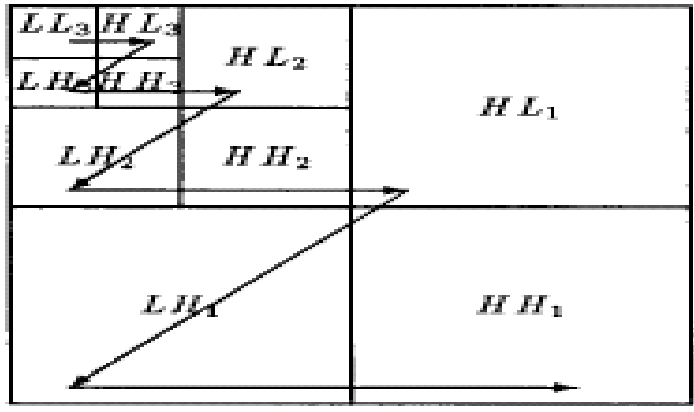

Figure 3. Scanning order of the sub-bands for encoding of the significance map

\subsection{Embedded Zero Tree Coefficient}

\section{Algorithm}

Embedded zero tree coding of wavelet coefficient was introduced by J.M.SHAPIRO in 1993. He designed the algorithm which was based on two hypothesis that "if a wavelet coefficient at a coarse level is insignificant with respect to threshold $\mathrm{T}$, then all wavelet coefficients of the same orientation the same spatial location at finer scale are likely to be insignificant with respect to T". The procedure begins with discrete wavelet transform to a given image .The image has been decomposed into three wavelets levels using filters "DAUBECHIES" filters. The algorithm codes contain two passes:-

(1) Dominant Pass

(2) Subordinate Pass

In the first pass there is a scanning of all the coefficients takes place on the dominant list using the current threshold $\mathrm{T}$. Then this dominant pass creates a list containing coefficients known as Subordinate list. Since the image which has been decomposed undergoes through another pass which is known as "subordinate pass".

Assign each coefficient one of four symbols:-

(a) Positive significant (PO)

(b) Negative significant (NE)

(c) Zero tree root $(\mathrm{ZR})$

(d) Isolated root (IZ)

PO means that the coefficient is significant relative to the current threshold $\mathrm{T}$ and positive.

NE means that the coefficient is significant relative to the current threshold $\mathrm{T}$ and negative.

$\mathrm{ZR}$ means that if the value of the coefficient is lower than the threshold $\mathrm{T}$ and has only insignificant descendants.

IZ means that if the absolute value of the coefficient is lower than the threshold $\mathrm{T}$ and has one or more significant descendants.

For each coefficient coded as significant (PO or NE), put its magnitude in the subordinate list and remove it from a dominant list .Any coefficient that is the descendant of a coefficient that already been coded as a zero tree root is not coded, since the decoder can deduce that it has a zero value. 
Coefficients found to be significant are moved to the subordinate list and there values in the original wavelet map are set to zero. Now in the subordinate pass set output as 1 or 0 for all coefficients on the subordinate list depending on whether the coefficient is in the upper or lower of the quantization interval. The coefficients in the sub-ordinate list are sorted in such a manner so as to enable the decoder to carry out the same sorting. In this manner all the coefficients are scanned and the process stops when the present threshold tends to become unity or at a stage based on any desired parameter like (MSE OR CR). The insignificant coefficients of the last sub-bands, which do not accept descendants and are not themselves descendants of a zero tree, are also considered to be zero tree.

\section{SIGNIFICANCE MAP ENCODING IN ZEROTREE CODING}

In this section the important aspect of low bit rate image coding is discussed. It has three basic components.

- Transformation

- Quantizer

- Data compression

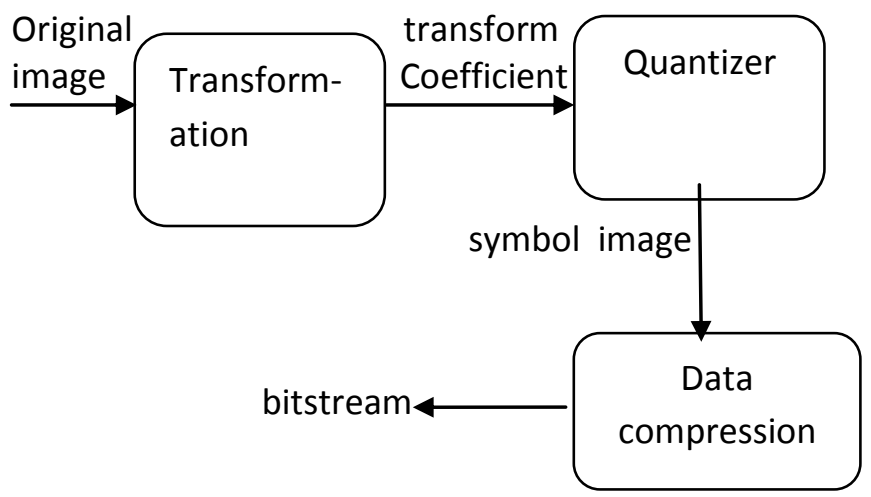

Figure 4 Encoding of Zero-tree coding

Original image passed through transformation to produce transform coefficient which is considered to be lossless and is decorrelated, then this transform coefficients are then quantized with the help of quantizer in which all information loss occurs here then it produce stream of symbols then data compression system takes the stream of symbols and attempts to lossless represent the data stream as efficiently as possible . In this a good transform should be able to de-correlate the image pixels, and provide energy compaction in transform domain so that very few quantized non-zero coefficients have to be encoded. Quantizer is a key component in wavelet image coding. Once quantization process is completed the last step of encoding is used to entropy coding or data compression system to achieve the rate (entropy rate) of the quantizer. To encoding the significance map it is useful to encode the sign of significant coefficients along with significant map. In this four symbols are used:-

- Zerotree root

- Isolated zero

- Positive significant

- Negative significant

In significance map coding last coefficient in the lowest resolution is the parent or ancestors of whole image. If a parent is insignificant in a low resolution image with reference to some threshold value then we assume that all its children are also insignificant.

Coefficients which are a root of a zerotree get a special sign (zt), signing that all their children are also neglected. Isolated zero(IZ) is a zero coefficient under the threshold but got at least one child that is not insignificant which means that coefficient is insignificant but has same significant descendants( for a given parent, the set of all the coefficients at all finer scales of similar orientation corresponding to same location). The sign code (positive/negative) is important for the progressive future.

\section{CHARACTERISTICS OF EMBEDDED CODER}

- It generates readable, compact and fast $\mathrm{C}$ and $\mathrm{C}++$ code for use on embedded processors on target rapid prototyping boards and microprocessors used in mass production.

- A Discrete Wavelet Transform (DWT) which provide compact multi-resolution representation of the image.

- EZW algorithm is based on successive approximation quantization and this facilitates the embedded algorithm and this successive approximation provides a compact multi-precision representation of the significant.

- Zerotree coding of wavelet coefficients.

- Adaptive arithmetic coding which provides a fast and efficient method for entropy coding string of symbols, and requires no pre-stored tables.

- Zerotree coding identifies the part of a tree that have all the DWT coefficients insignificant starting with a root.

- Zerotree allow the prediction of insignificant coefficient across scales to be efficiently represented as a part of exponentially growing trees.

\section{WAVELET AND MULTI RESOLUTION ANALYSIS}

Now a days wavelet transform is now used to compress, transmit and analyze many images. Unlike the Fourier transform, whose basis function are sinusoids, wavelet transforms are based on small waves called wavelets of varying frequency and limited duration and wavelet can be regarded as mathematic microscopes that permit one to zoom in and zoom out of images at multiple resolutions. In 1987, wavelets were first shown to be the foundation of a powerful approach to signal processing and analysis called multiresolution theory. Multi-resolution theory is considered with the representation and analysis of images at more than one resolution. This is shown by image pyramid which is a powerful, yet conceptually simple structure for representing images at more than one resolution. 


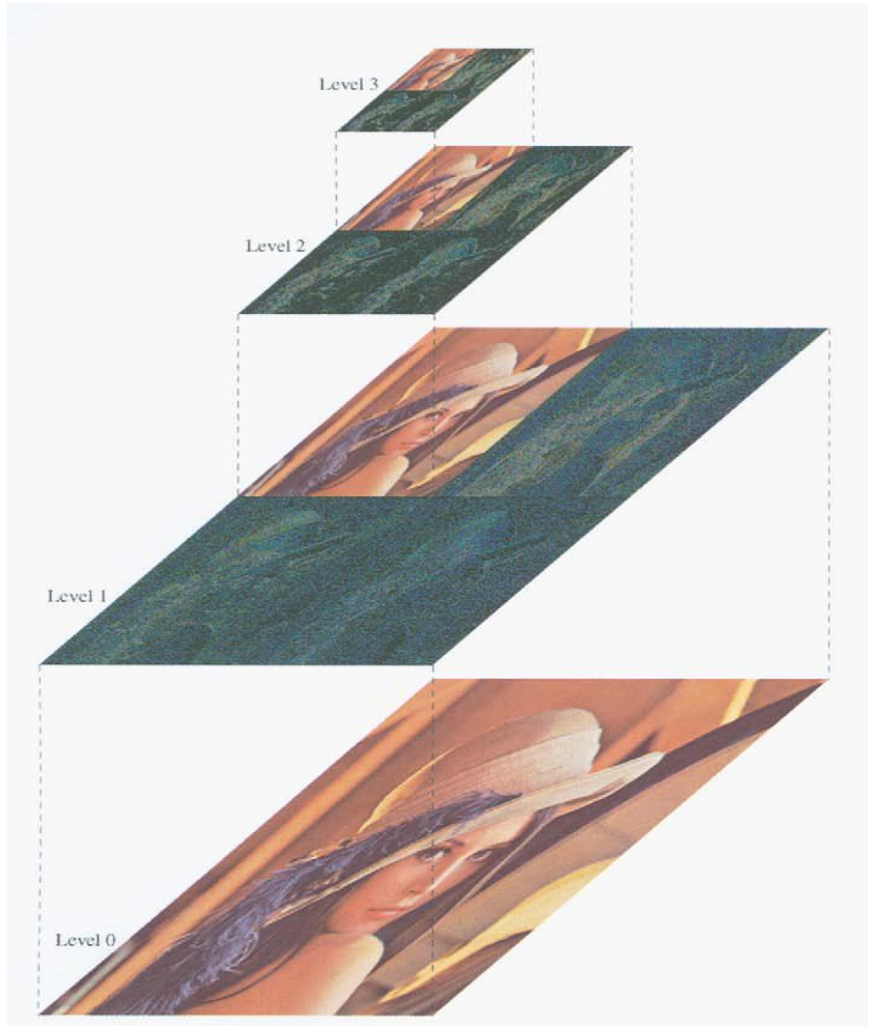

Figure 5. A three level hierarchy wavelet decomposition of the 512 : 512 color image LENA image. Level $1(512 \times 512)$ is the one leve wavelet representation of the original LENA at level 0; level 2(256 : 256)shows the one level wavelet representation of the low pass image a level 1 ; and level 3(128 x 128) gives the one level wavelet representation of the low pass image at level 2.

The base of this pyramid contains a high resolution representation of the image being processed and level 0 contains a low resolution representation of the image. In this figure as we move up the pyramid, both size and resolution decreases. Wavelets are the tools for decomposing signals such as images into increasing resolutions. As we consider more and more resolution layers we get more and more detailed look at the image. The main thing about the wavelet decomposition is that it enables the zooming characteristics at absolutely no cost in terms of excess redundancy. Base level $\mathbf{J}$ is of size $\mathrm{NxN}$ and level 0 is of size $1 \mathrm{x} 1$. If the objects are small in size or low in contrast, we normally examine them at high resolutions; if they are large in size or high in contrast a coarse view is all that is required. If both low and high contrast objects are present simultaneously, it can be advantageous to study at several resolutions.

\section{UNIT EMBEDDED ZERO TREE CODING}

\section{A. Unit Cell}

The proposed algorithm works on the smallest part of the image i.e. on the UNIT CELL.As we know that image is defined in terms of matrix, so in a similar way unit cell of an image can be defined in terms of matrix. Unit cell is the smallest possible matrix of an image generated from wavelet decomposed image which have the same level of decomposition as of the image. For a three level of sub image, the unit cell must be a square matrix, having the order of eight.

\section{B. Encoding Algorithm}

Similar, to the original algorithm, the proposed algorithm begins the encoding process, by the wavelet decomposition of the image, a desired number of times. The threshold is calculated for the entire image, once only and the same is used throughout the process. From the wavelet decomposed images, unit cells are generated. These unit cells are then coded using the original EZW coding algorithm. These units are then coded serially. For the embedded coding, the first dominant pass coefficients of all unit cells are combined followed by the next first sub-ordinate pass. The stream generated during dominant pass undergoes through a RUNLENGTH coder. This run-length coding is necessary because the generated bit-stream has a high order of redundancy, which arises due to the fact, that a single threshold value is utilized for each unit-cell. This run-length coding is followed by entropy coding. Similar to the original algorithm the bitstream generated during the sub-ordinate pass, needs not be run-length or entropy coded. This is followed by the next dominant and sub-ordinate pass and the process is repeated based on any desired image parameter. Once this parameter is obtained the encoder stops. The decoder must be aware of the order in which the unit cells were encoded, in order to be able to reconstruct the original image.

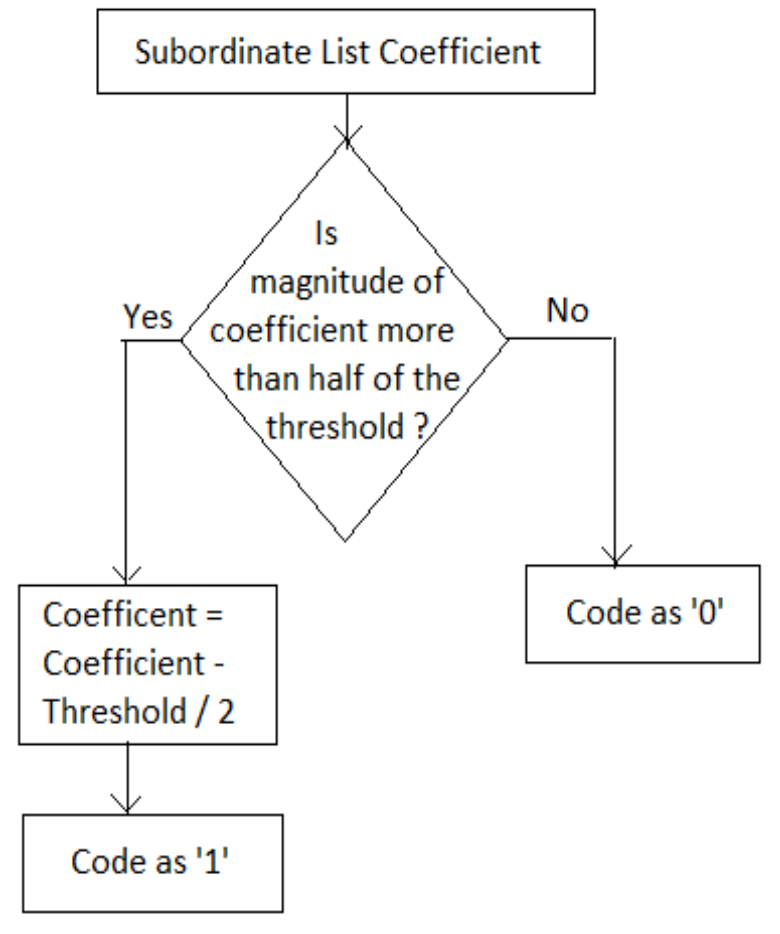

Figure 6. showing flowchart for encoding subordinate list coefficient during subordinate pass. This pass creates single set containing the symbols ' 1 ' and ' 0 '. 


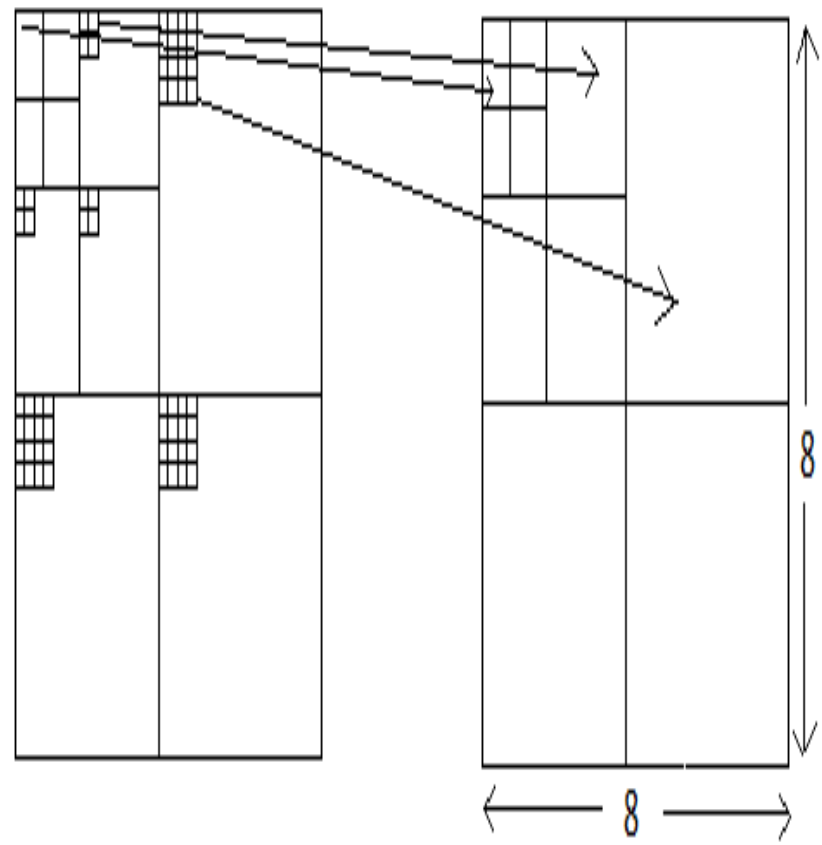

Figure 7 showing the formation of a unit cell from a wavelet decomposed sub-image. The arrow points from the sub-image to the unit cell. Similar relationship exist for elements in the LH, HH \& HL sub-bands also.

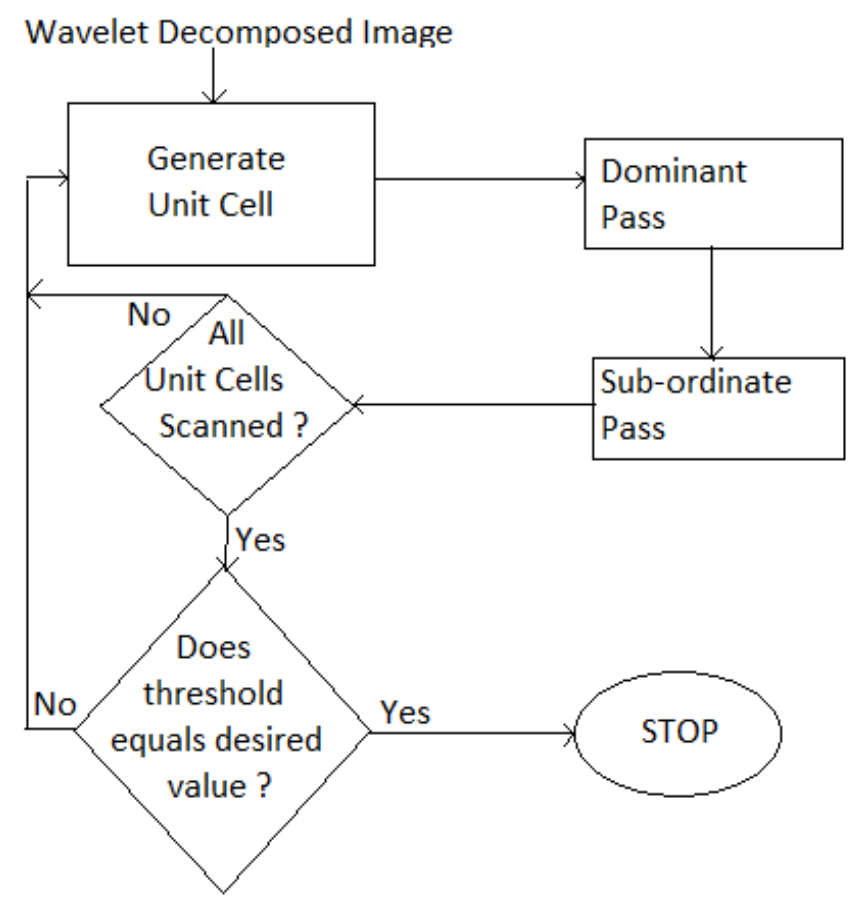

Figure 8. Showing the flowchart for the proposed algorithm

\section{COLOR MODEL}

The purpose of a color model (also called color space or color system) is to facilitate the specification of colors in some standard way. In essence a color model is a specification of a coordinate system where each color is represented by a single point. Most color models in use today are oriented either toward hardware such as for color monitors and printers. In terms of digital image processing, the hardware oriented models most commonly used in practice are the RGB (red, green, blue) model for color monitors.

\section{RGB COLOR MODEL}

The RGB color model is an additive color model in which red, green, blue are added together in various ways to reproduce a broad arrays of colors. The main purpose of this model is for sensing, representation and display of images in electronic systems such as televisions and computers. RGB is a devicedependent color model.

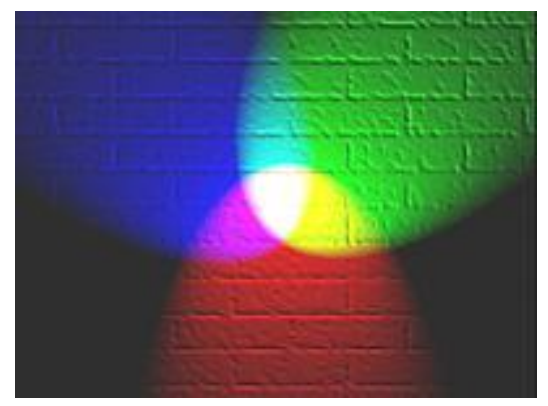

Figure 9 Representation of additive color mixing. Projection of primary color lights on a screen shows secondary colors; the combination of all three of red, green and blue in appropriate intensities makes white.

The name of the model comes from the three additives primary colors-red, green and blue. Image represented in the RGB color model contains three components images, one for each primary color. Typical input RGB devices are color TV and video cameras and output RGB devices are LCD, plasma etc.

\section{ADDITIVE PRIMARY COLORS}

To form a RGB color, three colored light beams (red, green and blue) must be superimposed (by emission from a black screen or by reflection from a white screen).

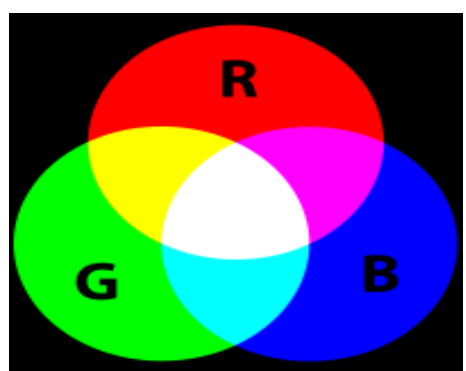

Figure 10 Set of primary colors

In this figure image is composed of primary and secondary RGB colors. The RGB color model is additive, which means the red, green and blue channels combine to create all the available colors in the system. When all the primary color values are the same, the result is neutral, or grayscale. For example, if all the three primary colors are zero percent or zero intensity of each image, the result is black. If all the primary colors are 100 percent or full intensity of each image, the result is white. When one of the components has strongest intensity, the color is a hue near the primary colors (red, green and blue). When two components have the same strongest intensity, then the color is a hue of secondary (shade of cyan, magenta or yellow). A secondary is formed by the sum of two 
primary colors of equal intensity and every secondary color is the complement of one primary color. When a primary color and its complementary secondary color are added together, the result is white.

\section{CHROMATICITY DIAGRAM}

Chromaticity diagram is useful for color mixing because a straight line segment joining any two points in the diagram defines all the different color variations that can be obtained by combining these two colors additively. To determine the range of colors that can be obtained from any three given colors in the chromaticity diagram, we simply draw connecting lines to each of the three color points, the result is a triangle. A triangle with vertices at any three fixed colors cannot enclose the entire color region. Use of the three primary colors is not sufficient to reproduce all colors; only within the color triangle defined by the chromaticities of the primaries can be reproduced by additive mixing of nonnegative amounts of those colors of light.

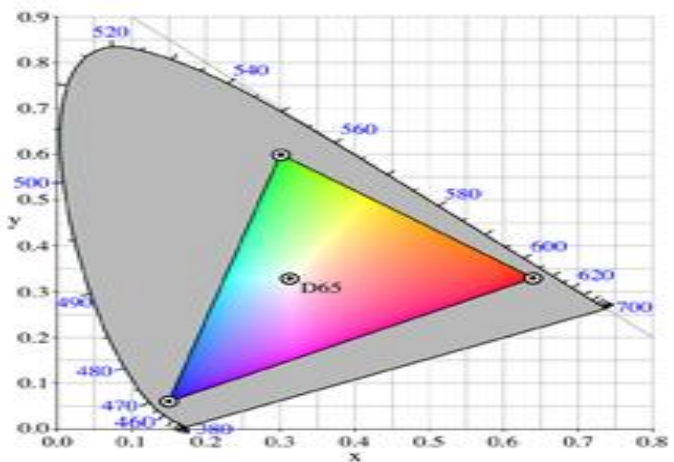

Figure 11 Chromaticity diagram (a set of primary colors,such as the RGB primaries, define a color triangle; only colors within this triangle can be reproduced by mixing the primary colors. Colors outside the color triangle are therefore shown here as gray).

\section{BREAKING OUT OF IMAGE COMPONENTS}

There are many ways to separate images into other types of components, including color components of light (red, green and blue). Separating images into components can offer advantages in making corrections, such as creating masks, setting up calculations, and converting images to black and white. Separation provides how images are composed, stored and viewed. Before digital imaging was developed, the method of doing separation of image into other components was to photograph the image three times, using a filter for each color. However this is achieved, the desired result is three grayscale images, which represent the red, green and blue components of the original image.RGB is another format for color images. It represents an image with three matrices of sizes matching the image format. Each matrix corresponds to one of the colors red, green or blue and gives an instruction of how much of each of these colors a certain pixel should use.In our work to find the encoding time for the colored image, the RGB components are first separated into R (Red), G (Green), and B (Blue) components. After this step they are separately encoded and the encoding time is calculated for each of them. At the final stage the encoding time is summed up.

\section{RESULTS AND DISCUSSION}

The result has been obtained for different test images having varying dimensions. The results were obtained for three levels of wavelet decomposition [7]. For the algorithm [1] the entire grayscale image was scanned and all three components were separated, after that the coding time was noted. In this furtherance the unit cells for the RGB image were composed and the same process was continued. In the reference paper [1] the encoding time was calculated for the gray scale image. Results for the RGB images have been shown in Table I. The results show a same considerable improvement in the coding time for the RGB image as for the grayscale image using the proposed algorithm [1] as compared to the original algorithm. This paper shows a similar advancement in the earlier unit embedded zerotree coding technique.

Table: 1 Comparison of coding time for the original and proposed algorithm (in seconds)

\begin{tabular}{|c|c|c|c|c|c|c|c|c|}
\hline \multirow{5}{*}{ Image } & \multicolumn{4}{|c|}{ EZW } & \multicolumn{4}{|c|}{ UEZW } \\
\hline & \multicolumn{4}{|c|}{$\begin{array}{c}\text { Dimensions } \\
(\text { Pixels } \times \text { Pixels })\end{array}$} & \multicolumn{4}{|c|}{$\begin{array}{c}\text { Dimensions } \\
(\text { Pixels } \times \text { Pixels })\end{array}$} \\
\hline & 32 & 64 & 128 & 256 & 32 & 64 & 128 & 256 \\
\hline & $\times$ & $x$ & $\times$ & $\times$ & $x$ & $x$ & $\times$ & $\times$ \\
\hline & 32 & 64 & 128 & 256 & 32 & 64 & 128 & 256 \\
\hline AL & 1.1630 & 4.9070 & 21.8820 & 116.9020 & 1.0170 & 4.0200 & 16.3760 & 63.3470 \\
\hline PEPPER & 2.2490 & 5.4560 & 24.5500 & 187.0490 & 1.1870 & 4.4750 & 17.3930 & 66.5430 \\
\hline KOALA & 1.3436 & 5.6670 & 25.0300 & 159.6180 & 1.1090 & 5.6420 & 17.2060 & 68.6420 \\
\hline
\end{tabular}




\begin{tabular}{crrrrrrrr} 
HUMAN-SKULL & 1.1700 & 1.1540 & 23.4940 & 112.7190 & 0.9670 & 0.9510 & 13.6970 & 54.8390 \\
NAINI & 1.3100 & 5.6630 & 24.5700 & 137.5140 & 1.1210 & 4.3370 & 17.1130 & 67.1890 \\
& 1.3300 & & & & & & & \\
FISH & & 5.6500 & 26.5790 & 140.5660 & 1.1600 & 4.3900 & 17.2100 & 69.6340 \\
\hline
\end{tabular}

\section{REFERENCES}

[1] A.S.Yadav (Asst.Professor) Electrical and Electronics Engineering,College of Engineering Roorkee, Roorkee,India -Unit embedded Zero-tree coding, International conference on advanced computing, communication and networks' 11 .

[2] Jerome M. Shapiro-Embedded image coding using zero trees of wavelet coefficients, IEEE Trans. Signal Process.41(12), (December 1993) 3445-3462.

[3] Module 5 -Embedded Wavelet Coding, Lesson 13, Wavelet Approach, Version 2, ECE IIT, Kharagpur .

[4] Vinay Kale, Shirish M. Deshmukh -Visually Improved Image Compression by Combining EZW Encoding with Texture Modeling using Huff. Encoderl, IJCSI International Journal of Computer Science Issues, Vol. 7 , Issue 3, No 11, May 2010,p. 28-38.

[5] V.S.Shingate (M.E.ELECTRONICS),Dr.T.R.Sontakke, Principal,S.G.G.S.E \&T Nanded, Prof. Dr. S.N.Talbar
B.A.T.U. Lonere- Still image compression using embedded zerotree wavelet coding.

[6] C.-Y. Teng, D. L. Neuhoff, J. A. Storer, and M. CohnQuadtree-guided wavelet image coding, in Proc. IEEE Data Compression Conf., 1996, pp. 406-415.

[7] Xiangang, EZW MATLAB Code [Online].Available: http://polyvalens.pagespersoorange.fr/clemens/download /ezwmatlab2.zip.

[8] Zixiang Xiong (Texas A\&M University), Kannan Ramchandran (University of California, Berkeley)Wavelet Image Compression copyright @ 2005 by Elsevier Academic Press.

[9] Xiaoyan Xu, School of Engineering, University of Guelph xux@uoguelph-Embedded Zerotree as image coding.

[10] G. J. Sullivan and R. L. Baker, -Efficient quadtree coding of images and F. Golchin and K. Paliwal, Quadtree based classification with arithmetic and trellis coded quantization for subband image coding, $\|$ in Proc. Int. Conf. ASSP, 1997, pp. 2921-2924 